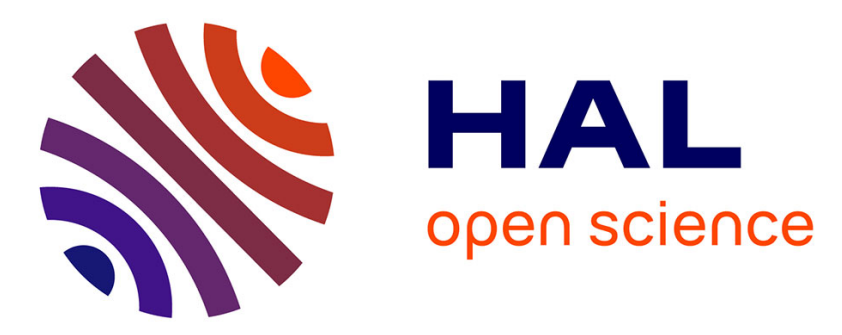

\title{
An Efficient OpenMP Loop Scheduler for Irregular Applications on Large-Scale NUMA Machines
} Marie Durand, Francois Broquedis, Thierry Gautier, Bruno Raffin

\section{To cite this version:}

Marie Durand, Francois Broquedis, Thierry Gautier, Bruno Raffin. An Efficient OpenMP Loop Scheduler for Irregular Applications on Large-Scale NUMA Machines. International Workshop on OpenMP (IWOMP), Sep 2013, Canberra, Australia. pp.141-155, 10.1007/978-3-642-40698-0_11. hal-00867438

\section{HAL Id: hal-00867438 https://hal.inria.fr/hal-00867438}

Submitted on 29 Sep 2013

HAL is a multi-disciplinary open access archive for the deposit and dissemination of scientific research documents, whether they are published or not. The documents may come from teaching and research institutions in France or abroad, or from public or private research centers.
L'archive ouverte pluridisciplinaire HAL, est destinée au dépôt et à la diffusion de documents scientifiques de niveau recherche, publiés ou non, émanant des établissements d'enseignement et de recherche français ou étrangers, des laboratoires publics ou privés. 


\title{
An Efficient OpenMP Loop Scheduler for Irregular Applications on Large-Scale NUMA Machines
}

\author{
Marie Durand $^{1}$, François Broquedis ${ }^{2}$, Thierry Gautier ${ }^{1}$, and Bruno Raffin ${ }^{1}$ \\ ${ }^{1}$ INRIA, ${ }^{2}$ Grenoble Institute of Technology \\ MOAIS Team, Computer Science Laboratory of Grenoble, France \\ marie.durandeinria.fr, françois.broquedis@imag.fr, \\ thierry.gautier@inrialpes.fr, bruno.raffineinria.fr
}

\begin{abstract}
Nowadays shared memory HPC platforms expose a large number of cores organized in a hierarchical way. Parallel application programmers struggle to express more and more fine-grain parallelism and to ensure locality on such NUMA platforms. Independent loops stand as a natural source of parallelism. Parallel environments like OpenMP provide ways of parallelizing them efficiently, but the achieved performance is closely related to the choice of parameters like the granularity of work or the loop scheduler. Considering that both can depend on the target computer, the input data and the loop workload, the application programmer most of the time fails at designing both portable and efficient implementations. We propose in this paper a new OpenMP loop scheduler, called adaptive, that dynamically adapts the granularity of work considering the underlying system state. Our scheduler is able to perform dynamic load balancing while taking memory affinity into account on NUMA architectures. Results show that adaptive outperforms state-of-the-art OpenMP loop schedulers on memorybound irregular applications, while obtaining performance comparable to static on parallel loops with a regular workload.
\end{abstract}

Keywords: OpenMP, NUMA, loop scheduling, runtime systems.

\section{Introduction}

Large-scale multicore platforms are commonly used by the HPC community. They expose a constantly-growing number of cores organized in a hierarchical way, leading to large-scale NUMA machines. To exploit them at their full potential, the application programmer needs to express massive fine-grain parallelism while taking memory affinity into account. Applications exposing irregular workloads are really difficult to execute efficiently on such platforms, as they require to deal with both load balancing and memory locality.

OpenMP [17], the de-facto standard for shared memory parallel programming, provides the programmer with high-level constructs to ease the parallelization of serial applications. The parallel loop certainly remains the most widely used OpenMP construct, allowing to easily parallelize loops with independant iterations. The end-user can control the way loop iterations are assigned to OpenMP threads by invoking OpenMP loop schedulers. Choosing the best scheduler for a specific parallel loop can be a difficult task to perform in a portable way [1]. The application programmer is also responsible for defining the granularity of work within the loop, using the chunk_si ze clause. 
While being designed to tackle loops with irregular workloads, the OpenMP dynamic scheduler suffers from two main issues on large-scale NUMA machines.

First, defining a chunk size from the application level that achieves both high and portable performance has never been so difficult. Indeed, parallel loops with big chunks may suffer from load imbalance, while the ones with smaller chunks are more sensitive to runtime-related overheads which are getting more and more noticeable as the number of cores per NUMA node increases.

Second, traditional techniques to increase the performance of memory bound applications, like guiding the data allocation on the different NUMA nodes of the platform, are useless using dynamic scheduling, as the assignment of loop iterations to OpenMP threads is non-deterministic.

We introduce in this paper a new loop scheduler, called adaptive, that outperforms state-of-the-art loop schedulers when executing memory bound irregular applications. In particular, our loop scheduler:

1. dynamically adapts the granularity of work within parallel loops according to the machine resources utilization,

2. relies on data placement information to guide load balancing on NUMA platforms.

The remainder of the paper is organized as follows. We first describe the related work on loop scheduling over NUMA architectures in section 2. Then we introduce the adaptive loop scheduler and the way we implemented it inside the LIBGOMP library in sections 3 and 4 . Eventually, we report the performance we obtained on several benchmarks and applications in section 5 before concluding.

\section{Related Work}

Many research projects have been carried out to improve execution of OpenMP applications on NUMA machines.

The HPCTools group at the University of Houston has been working in this area for a long time, proposing compile-time techniques that can help improving memory affinity on hierarchical architectures like distributed shared memory platforms [13]. Huang et al. [10] proposed OpenMP extensions to deal with memory affinity on NUMA machines, like ways of explicitly aligning tasks and data inside logical partitions of the architecture called locations. While the proposed extension is interesting to deal with regular memory-bound applications, it does not tackle the problems induced by irregular workloads.

Olivier et al. $[16,15]$ introduced node-level queues of OpenMP tasks, called locality domains, to ensure tasks and data locality on NUMA systems. The runtime system does not maintain affinity information between tasks and data during execution. Data placement is implicitly obtained considering that the tasks access memory pages that were allocated using the first-touch allocation policy. The authors thus ensure locality by always scheduling a task on the same locality domain, preventing application programmers to experiment with other memory bindings.

The INRIA Runtime group at the University of Bordeaux proposed the ForestGOMP runtime system [2] that comes with an API to express affinities between 
OpenMP parallel regions and dynamically allocated data. ForestGOMP implements load balancing of nested OpenMP parallel regions by moving branches of the corresponding tree of user-level threads on a hierarchical way. Memory affinity information is gathered at runtime and can be taken into account when performing load balancing. Our work extends this approach to deal with parallel loops while ensuring load balancing in a different way.

Mahéo et al. [12] used similar techniques to speed up hybrid MPI/OpenMP synchronizations on hierarchical architectures, including NUMA machines. Both our work and theirs build upon the same concepts and can be stated as complementary.

Subramanian and Eager [18] introduce an affinity loop scheduler for unbalanced workload. They study iterative applications involving series of parallel loops in which "the execution times of any particular iteration do not vary widely from one execution of the loop to the next"[18]. Their proposition is based upon a two-phase algorithm: first, the iterations are equally divided between the processors; then the scheduler dynamically adapts the workload by making idle processors steal a constant fraction $(1 / P)$ of the remaining iterations from occupied ones. In [21], Yong et al. extends the work of Subramanian and Eager by providing new ways of adapting the workload considering an history of previous executions of a particular parallel loop.

Taking advantage of the temporal coherency of the simulation is a very interesting idea but cannot be used in all situations. For instance, it would not be effective on applications involving several parallel loops with varying workloads, like the PMA application we used to evaluate our adaptive loop scheduler. The first phase of our scheduling strategy is similar to the one introduced by Subramanian et al., as adaptive equally pre-distributes the loop iterations over the processors, which is a compromise between balancing the workload of the loop and preserving the affinity across several executions of the same loop. However, the second phase of our algorithm is different from the one implemented by Subramanian's adaptive loop scheduler. Indeed, adaptive relies on a work-stealing algorithm [8] in which idle processors steal half of the remaining iterations from a randomly-selected victim. With such an approach, our adaptive loop scheduler does not require to maintain a global view of the workloads associated to each processor, unlike proposition [18].

\section{Introducing the Adaptive Loop Scheduler}

The OpenMP programmer can rely on two main loop schedulers to specify the way loops iterations should be assigned to threads. The first one, called static, statically assigns fixed portions of work in a round-robin fashion. This scheduler behaves well on loops with a regular workload and is often used in the context of NUMA architectures, along with the first-touch allocation policy, to maximize memory locality. The second one, called dynamic, makes OpenMP threads steal fixed portions of work from a centralized queue. This scheduler behaves better than static on loops with an irregular workload. However, dynamic is seldom used on NUMA architectures because of its non-deterministic behavior, preventing the programmer from controlling memory locality. 
The loop scheduler we propose goes beyond those two, providing ways of balancing the load of irregular loops while respecting memory locality. This section first introduces the main scheduling algorithm provided by our adaptive loop scheduler before presenting the way we extended it to deal with memory locality on NUMA machines.

\subsection{Designing an OpenMP Loop Scheduler with Adaptive Granularity}

Dealing with irregular parallel applications requires efficient runtime-level functionalities to perform dynamic load balancing with the lowest overhead possible. OpenMP application programmers can rely on the dynamic loop scheduler to execute loops with irregular workload, as long as they manage to specify a chunk size that achieves good performance. Indeed, a too small chunk size will increase the time spent inside the runtime system, while a too coarse chunk size will limit the potential parallelism and the ability to balance the work load.

We adopted a different approach implementing our adaptive scheduler. We relieve the application programmer from statically deciding the granularity of work which can lead to non-portable solutions. Instead, we consider work-stealing as an oblivious technique to dynamically balance the load on the threads of the corresponding OpenMP parallel region.

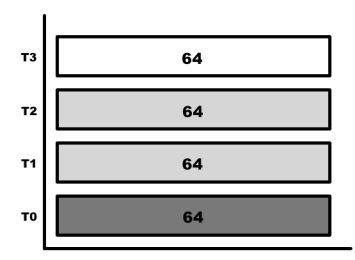

(a) Initial distribution of the iteration space.

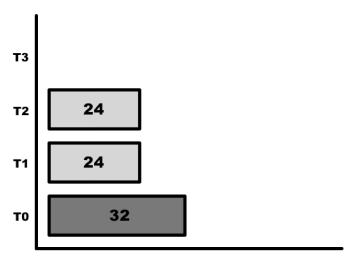

(b) $\mathrm{T} 3$ has completed its range and starts stealing.

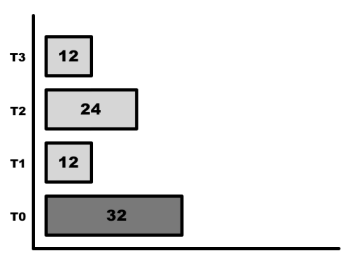

(c) T3 stole half of the remaining iterations of $\mathrm{T} 1$.

Fig. 1: Illustration of adaptive loop scheduling on a 256-iterations loop with irregular workload executed on 4 threads. Darker color means higher workload.

We broke the vision of centralized work used by the state-of-the-art OpenMP loop scheduler to introduce a per-thread data structure describing the range of iterations assigned to each thread. Figure 1 illustrates the behavior of our adaptive scheduler on a synthetic example. Considering a loop of imax iterations executed by nthr threads, the scheduler first assigns imax / nthr iterations to each thread, like presented on figure 1a. This initial behavior allows our scheduler to achieve performance that is comparable to static on loops with regular workload. Even if every thread has the same number of iterations to execute here, the associated workload is different: the 64 iterations of thread T3 have shorter execution times than the ones assigned to T2 for example. This leads to load imbalance: at some point of the execution of the loop, thread T3 will be starving like showed on figure 1b. T3 will then trigger adaptive's work-stealing algorithm which steals half of the remaining iterations of a loaded thread. 


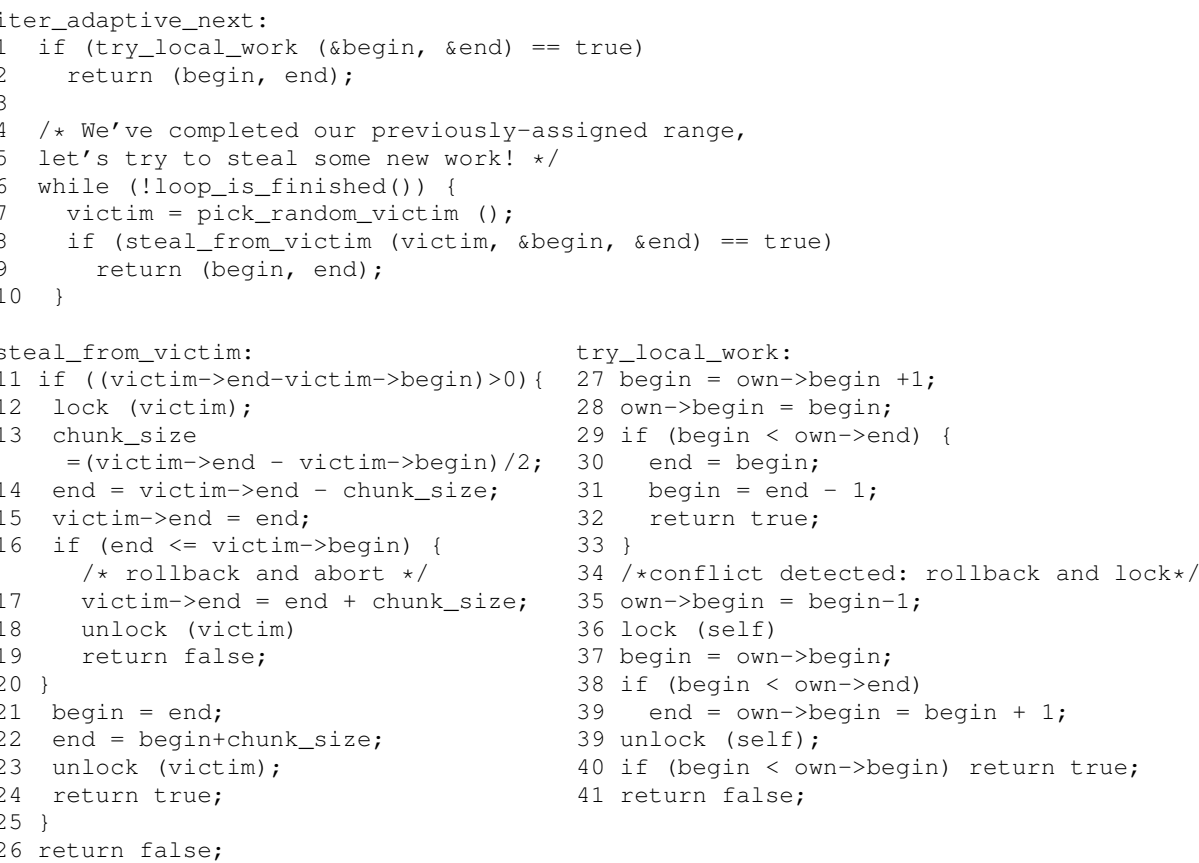

Fig. 2: Pseudo-code of the adaptive loop scheduler. The implementation extends the THE protocol by stealing more than one item at each steal operation.

The algorithm ${ }^{1}$ called to select the next chunk of iterations to execute is summarized on figure 2. Most importantly, our approach deals with dynamic per-thread chunk sizes, as shown on line 13 of this pseudo-code. The amount of work a thread will steal depends on the amount of work its victim still has to execute, unlike the dynamic scheduler in which the chunk_size is statically defined and cannot change during execution.

Unlike $[18,21]$ in which a constant fraction of the work $(1 / P)$ is removed from the most loaded processor, the random selection of the work-stealing algorithm does not suffer from maintaining the global state of the workloads associated to processors. Moreover, it is possible to derive theoretical performance guarantee of scheduling parallel loop with work stealing [20]. Frigo et al. [8] introduced two main metrics to model the performance of work-stealing-based algorithms: the work $W$, i.e. the time to sequentially execute the loop, and the depth $D$, also called the critical path, i.e. the execution time on an infinite number of processors.

Considering these metrics, the average completion time of the parallel loop is $O(W / P+D)$. If the work is $W=\sum_{i=0}^{n-1} w_{i}$, where $w_{i}$ is the work associated to the $i$-th iteration, then $D=O\left(\log n+\max \left\{w_{i}\right\}\right)$.

\subsection{Extending the Adaptive Scheduler to Deal with Locality}

Ensuring memory locality is crucial to achieve good performance on NUMA architectures. We extended the adaptive scheduler in order to benefit from shared memory

\footnotetext{
${ }^{1}$ If the memory consistency is not sequential, memory barriers have to be inserted between lines 15-16 and 28-29.
} 


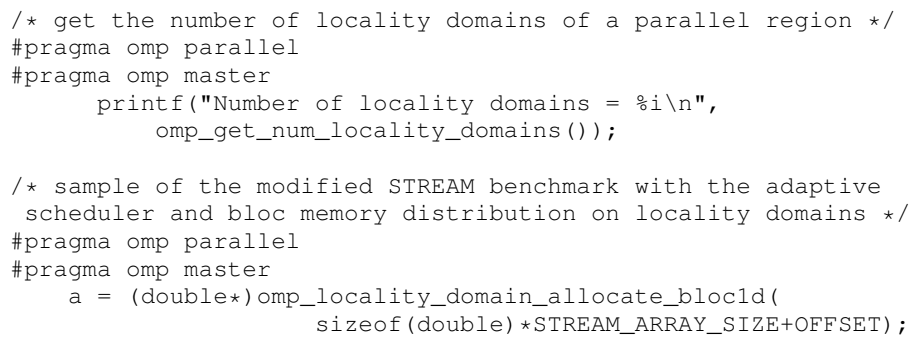

Fig. 3: Code sample using our extended OpenMP runtime APIs.

banks of NUMA multicore machines. This is done at two levels. First, we make the cores attached to the same memory bank work on contiguous iterations. This step is useful to abstract the OS identification of cores that may not be contiguous on a NUMA node. adaptive makes idle threads steal work from cores that belong to the same NUMA node. Thanks to this strategy, a successful steal will hopefully reduces the number of remote memory transfers. Moreover, this local steal strategy may not be enough to balance the workload among all the cores. That is why, if the number of unsuccessful steal operations reaches a threshold, the idle thread emits a steal request to a victim randomly selected over the whole machine.

The second feature is to provide ways of distributing the application data over the NUMA nodes. Our extension of the libGOMP library comes with APIs to distribute data as proposed in MAMI [4], implementing bloc and bloc-cyclic data distributions as runtime extensions. For now, we only support data distribution within parallel regions where the participating threads are bound to cores, for example using the GOMP_CPU_AFFINITY environment variable. As in [10,16, 15], we refer to NUMA nodes as locality domains.

omp_get_num_locality_domains () : returns the number of locality domains holding threads from the current OpenMP parallel region.

omp_get_locality_domain_num() : returns the locality domain of the running thread. omp_locality_domain_allocate_bloc1dcyclic (size, blocsize) : allocates an array of size bytes in a blocsize-cyclic distribution over the locality domain of the parallel region.

omp_locality_domain_allocate_bloc1d (size) : allocates an array of si ze bytes using a bloc distribution over the locality domain of the parallel region.

These routines performs allocation following the OS constraints: sizes are rounded up to a multiple of page size. If the OS does not support NUMA allocation routines, the implementation triggers calls to the libc's malloc function.

Figure 3 illustrates the use of the runtime APIs we propose. In order to allow reuse of memory mapping across parallel regions, we ensure that the $i-t h$ thread of a parallel region will be bound to the same core across parallel regions if and only if the following parallel regions have the same size and are nested in the same parallel region or are at the top level. 


\subsection{Discussion}

Defining the best granularity of work is certainly one of the most difficult challenge a parallel application programmer has to face to exploit HPC platforms at their full potential. For example, the best chunk_size for a specific OpenMP loop may depend on the target architecture and the input data of the parallel application. In other words, application programmers have to consider the underlying system state to specify the granularity that will achieve the best performance. This is an old problem for the OpenMP community: defining the right number of threads, the right level of nested parallel regions and the right chunk size for parallel loops are a few examples of the many crucial steps to achieve good performance and scalability.

The addition of tasks to the OpenMP standard provides new ways of expressing parallelism with a finer granularity. One can consider tasks as another way of dealing with irregular workload, as tasks can move from one OpenMP thread to another to perform load balancing. However, tasks will not solve the problem of granularity, as defining the right number of tasks can be challenging, as studied in our previous work [3].

Our proposal introduces a runtime-level approach to deal with granularity and has been implemented inside a loop scheduler. The same approach could be applied to task parallelism as well, considering ways of splitting OpenMP tasks when necessary. Our group has carried out research in this context [19] that could be extended to OpenMP. The application programmer could provide functions to split a running task into smaller ones, similarly to the way our adaptive loop scheduler splits ranges of iterations. This idea was applied to more general iterative algorithms where dependencies may exist between iterations [20].

\section{Implementation Details on Extending libGOMP with Adaptive Loop Scheduling}

We implemented our adaptive loop scheduler inside the original LIBGOMP library that comes with GCC 4.6.2. Our loop scheduler can be experimented with parallel loops stated as schedule (runtime) by setting the OMP_SCHEDULE environment variable to "adaptive, chunk_size" before running the application. This allows us to experiment with our proof-of-concept implementation without modifying the compiler.

The implementation (figure 2) of the stealing mechanism used in the adaptive loop scheduler is greatly inspired from Cilk's THE algorithm [8] designed to limit the perturbation of the serial execution from stealing-related overheads. Unlike other OpenMP loop schedulers, adaptive uses a per-thread data structure describing the range of iterations assigned to the considered thread. This structure basically contains the boundaries of this range ([begin, end)) and an atomic-based lock used to synchronize the stealing thread and its victim. Each thread pops chunk_size iterations to execute out of its own range (begin $+=$ chunk_size), until there are no more iterations left $($ begin $==e n d$ ). Stealing a range of iterations from a busy thread is simply a matter of shrinking the end bound of the victim's data structure down to end $-N, N$ being the number of iterations we want to steal. The THE algorithm uses an optimistic approach to minimize the need for a thread to lock its own data structure on a pop operation. This can be done 


\begin{tabular}{c|c|c|c|c|c|c|c|c} 
chunk size & 1 & 2 & 4 & 8 & 16 & 32 & 64 & 128 \\
\hline \hline static & 30.44 & 28.20 & 25.97 & 25.03 & 24.40 & 24.50 & 24.18 & 24.50 \\
\hline dynamic & 1328.43 & 594.30 & 232.61 & 75.43 & 36.29 & 35.20 & 34.02 & 33.21 \\
\hline guided & 77.86 & 69.49 & 55.55 & 45.47 & 42.90 & 43.16 & 58.66 & 30.54 \\
\hline adaptive & 55.92 & 50.74 & 48.26 & 47.72 & 47.69 & 47.97 & 48.90 & 49.44 \\
\hline \hline adaptive (no steal) & 30.29 & 27.48 & 25.67 & 24.48 & 25.21 & 24.48 & 24.16 & 23.23
\end{tabular}

Table 1: Overhead measured by EPCC benchmark (in $\mu s$ ) of the adaptive loop scheduler versus static, dynamic and guided on the AMD48 platform.

by detecting conflicting accesses to the same data structure, by comparing the value of end before and after the pop operation. If the value has changed, someone accessed the data structure during the pop: the thread will then undo this last pop and acquire the lock before trying again. Such implementation greatly minimizes the overhead added to threads performing local work (Cilk's work first principle [8]).

The memory binding routines rely on libNUMA and the mbind system call. The current prototype was developed on Linux. libGOMP maintains a pool of threads (gomp_thread and gomp_thread_pool) attached to each parallel region. We extended the data structures in order to maintain simple per-thread NUMA-related information, like the core id, the NUMA node id and a list of threads per NUMA node that can be used by the adaptive scheduler to select a victim. Based on this information, the scheduler initializes of per-loop data structure to balance the iterations over the NUMA nodes taking the number of cores per NUMA node into account.

\section{Performance Evaluation}

We conducted our experiments on two different ccNUMA configurations.

The first one holds 8 AMD Magny Cours processors for a total of 48 cores. Each core has access to $64 \mathrm{~KB}$ of L1 cache, $512 \mathrm{~KB}$ of L2 cache. Both L1 and L2 caches are private, while $\mathrm{L} 3$ cache is shared between the 6 cores of a processor. This configuration provides a total of $256 \mathrm{~GB}$ ( $32 \mathrm{~GB}$ per NUMA node) of main memory. We will refer to this configuration as AMD48 in the following of the paper.

The second configuration holds 12 groups containing two Intel Sandy Bridge processors each for a total of 192 cores. $32 \mathrm{~GB}$ of main memory is attached to each socket, for a total of $768 \mathrm{~GB}$. Inter-groups communications use the SGI NUMAlink technology, while standard Intel QuickPath interconnect provides inner-group communications. We will refer to this configuration as Intel192 in the following of the paper.

All experiments were performed with the libGOMP library distributed with GCC 4.6.2.

\subsection{EPCC: Overhead of the Adaptive Loop Scheduler}

The EPCC benchmark [5] reports runtime-related overheads when performing OpenMP loop scheduling with respect to the corresponding serial execution. The measured overheads of the four loop schedulers are reported in table 1 for different chunk sizes. A 
larger chunk size implies less calls to the runtime and thus a smaller overhead. Reported measures reported represent the average performance over 10 runs. The libGOMP implementation of both the dynamic and the guided schedulers suffer from a high overhead for the three smallest chunk size values tested in this experiment.

The adaptive scheduler adds an extra overhead to $25 \mu \mathrm{s}$ with respect to the static scheduler. By disabling the steal operations from the adaptive scheduling algorithm, we are able to provide finer estimation of the overheads. The performance of this modified scheduler, named adaptive (no steal) in the table, reports no overhead induced by the initial work distribution over the static scheduler. We can thus infer that an extra $25 \mu s$ includes the overheads of the work-stealing operations and the detection of the termination.

Obviously, our code is not as optimized as the other scheduler implementations from libGOMP. We will add some optimizations (memory alignment of data structures, lazy initialization) to reduce overheads, and we believe that those may improve the performance of all libGOMP schedulers as well.

\subsection{STREAM: Impact of the Memory Hierarchy}

The STREAM benchmark [14] measures the maximal achievable bandwidth over four memory bound kernels (copy, scale, add and triad). We evaluated the behavior of the static and the adaptive schedulers with two memory allocation strategies: a firsttouch strategy and an explicit bloc distribution of the arrays over the 8 NUMA nodes of the AMD48 platform using the API presented in section 3.2. The memory per array is $150.0 \mathrm{MB}$ and the number of iterations is set to 500 . Measures are reported in table $4 \mathrm{a}$.

\begin{tabular}{|c|c|c|c|c|}
\hline & \multicolumn{2}{|c|}{ static } & \multicolumn{2}{c|}{ adaptive } \\
\hline & $\begin{array}{c}\text { first- } \\
\text { touch }\end{array}$ & bloc & $\begin{array}{c}\text { first- } \\
\text { touch }\end{array}$ & bloc \\
\hline Copy & 6.9 & 6.9 & 6.4 & 6.8 \\
Scale & 6.7 & 6.8 & 6.2 & 6.7 \\
Add & 7.2 & 7.4 & 6.8 & 7.3 \\
Triad & 6.9 & 7.5 & 6.6 & 7.4 \\
\hline
\end{tabular}

(a) Achieved bandwidth $(\mathrm{GB} / \mathrm{s})$ reported by the STREAM benchmark for the static and adaptive loop schedulers.

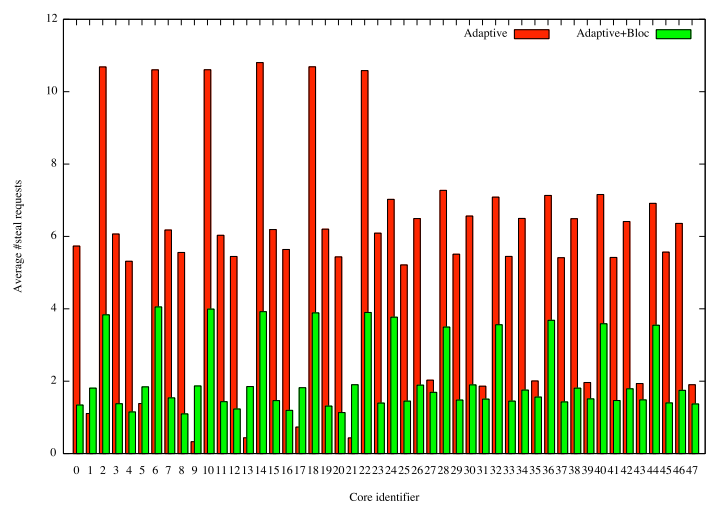

(b) Number of steal operations with the adaptive scheduler with and without bloc memory allocation.

Fig. 4: Performance evaluation of the STREAM benchmark on the AMD48 platform.

On the Triad kernel, the bloc allocation strategy increases the performance of at most $7 \%$ with the static scheduler and of at most $12 \%$ with the adaptive scheduler. The 
two schedulers reach comparable performances on this highly regular benchmark, with a slight advantage for static over adaptive.

This difference comes from the steal operations performed by the adaptive scheduler. Figure $4 \mathrm{~b}$ shows the average number over 500 iterations of successful steal requests per core. We can see that the adaptive scheduler with the NUMA-aware extension taking the memory distribution into account helps reducing the number of steal operations.

On this memory-intensive benchmark, adaptive is able to reach performances that are similar to static as the load balancing strategy first conside the cores from the same NUMA node to perform work-stealing, thus favoring memory locality on such architectures.

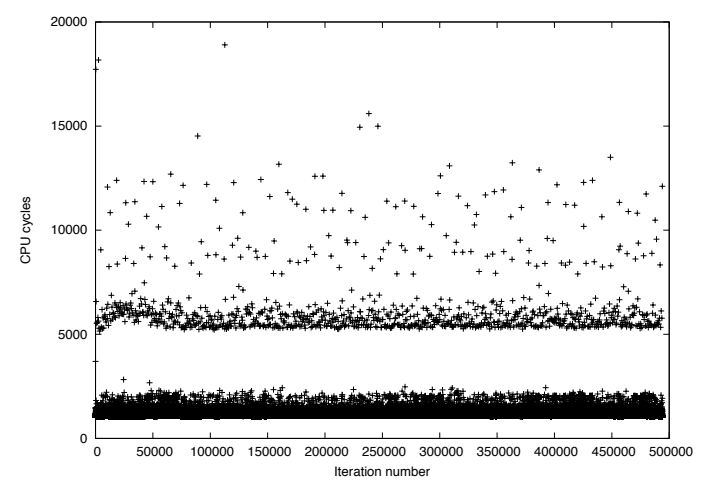

(a) Execution time, in CPU cycles, of each iteration of the kmeans kernel main loop on a single core of the Intel192 platform.

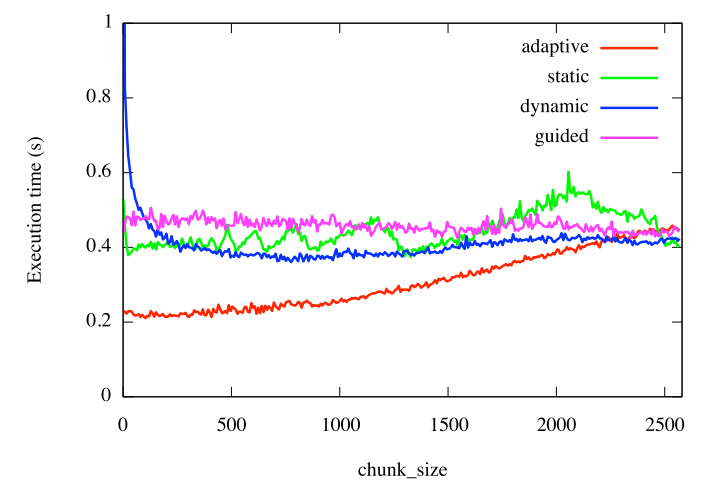

(b) Average performance of different OpenMP loop schedulers for varying values of chunk_size on the Intel192 platform.

Fig. 5: Performance evaluation of the K-Means benchmark on the Intel192 platform. 


\begin{tabular}{|c|c|c|c|c|c|}
\hline $\begin{array}{c}\text { Time in } \\
\text { ms }\end{array}$ & static & $\begin{array}{c}\text { dynamic } \\
\text { chunk=1 }\end{array}$ & $\begin{array}{c}\text { dynamic } \\
\text { chunk=3000 }\end{array}$ & guided & adaptive \\
\hline \hline numactl & 17.8 & 57.4 & 12.1 & 15.2 & 11.6 \\
\hline bloc distribution & 16.3 & 57.2 & 14.2 & 14.9 & 6.95 \\
\hline
\end{tabular}

Table 2: Comparison of the four loop schedulers on PMA on the AMD48 platform.

\subsection{K-Means: Benefits of Adaptive Granularity for OpenMP Loops}

We evaluated the adaptive loop scheduler with the OpenMP version of the K-Means kernel coming from the Rodinia benchmark suite [6]. This kernel implements a clustering algorithm commonly used by data-mining applications. Its parallel implementation involves an OpenMP parallel loop with an irregular workload.

Figure 5a reports the execution times of each one of the 494020 iterations of this loop executed on a single core of the Intel192 platform. We can distinguish at least two main classes of iterations on this figure with different execution times, but we can only consider this as a rough source of information, as the execution times of the same iterations may vary when executing them in parallel, depending for example on the capacity of threads to efficiently communicate through shared cache memory.

The results obtained running this kernel with the adaptive scheduler confirms KMeans can benefit from dynamic load balancing. Figure $5 \mathrm{~b}$ shows a performance comparison between the adaptive, static, dynamic and guided schedulers on the K-Means kernel. We experimented with different values for the chunk_size clause of the for loop. These tests were executed on the 192 cores of the Intel192 platform. We tested every value of chunk_size from 1 to 2574 , corresponding to imax / nthr here. The best performance is achieved by our scheduler. adaptive performs especially well for executions with small chunk sizes, as they offer more options to perform load balancing. We can also note that, even if the workload of this kernel is irregular, the best performance of the dynamic scheduler can almost be achieved by static for a tuned value of chunk_size.

\subsection{PMA: Dealing with Both Load Balancing and Locality}

We applied our adaptive loop scheduler to a practical situation in physical simulations considering elements of a 3D space that evolve with respect to physical laws. Maintaining these elements ordered is a key factor to improve memory efficiency as elements are likely to interact with their neighbors [11].

The Packed Memory Array (PMA) [7] data structure has been proposed to help maintaining its elements ordered in an efficient way. This sparse data structure was designed to reduce the amount of memory movement induced by reordering operations.

We focus on the loop that handles both the detection of the moving elements and their copy in a dense array. In real applications, the workload gets irregular since some parts of the physical space go through a lot of changes while others report only a few changes. We extracted actual change distribution from a memory-intensive fluid simulation [9] ran with 2900000 elements.

In this code, the data structure is initialized from reading sequentially a file. Without major rewrite of the initialization phase, it is not possible control the 
affinity with the simple first touch strategy as OpenMP standard preaches it. Table 2 reports average times for each of the four loop schedulers with two memory distribution strategies. The first strategies, called numactl in the table, distributes memory pages in a round robin fashion among the NUMA nodes using numact l --interleave. The second strategy leads to a bloc distribution using the API omp_locality_domain_allocate_blocld presented in section 3.2.

When the array is distributed with the bloc distribution, contigous elements in the array are mostly on the same NUMA node. Even with this distribution, the static scheduler does not reach the best time, which illustrates the irregularity of the application. The dynamic and guided schedulers are able to improve performance but without control of the locality while workload is balanced. The adaptive scheduler obtains the best times for the two memory distribution strategies. This is due to a good compromise between a good balance of iterations to control affinity and a dynamic balance of the workload.

The plots in figures 6,7 and 8 correspond to execution where the memory is bound using bloc distribution strategy among the NUMA nodes.

Figures $6 \mathrm{a}$ and $6 \mathrm{~b}$ report a per-core execution using the libGOMP dynamic and guided schedulers with a bloc data distribution over the NUMA nodes. The "compute" (green) part of the graph represents the time spent, in CPU cycles, during the execution of the loop body. The "schedule" part represents the time spent to perform the required computations, apply the scheduling decisions and wait until the loop completion.

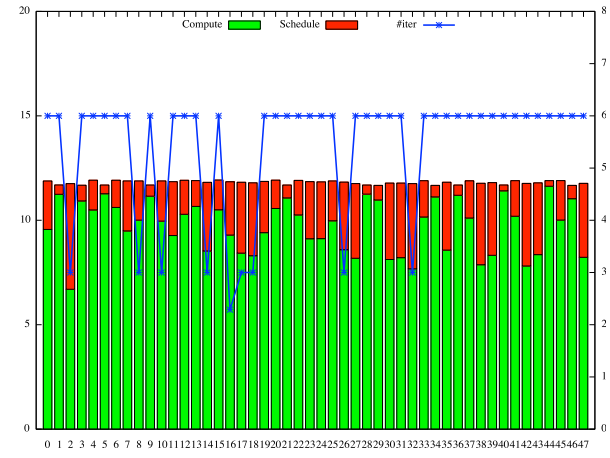

(a) Dynamic scheduler, chunk size $=3000$

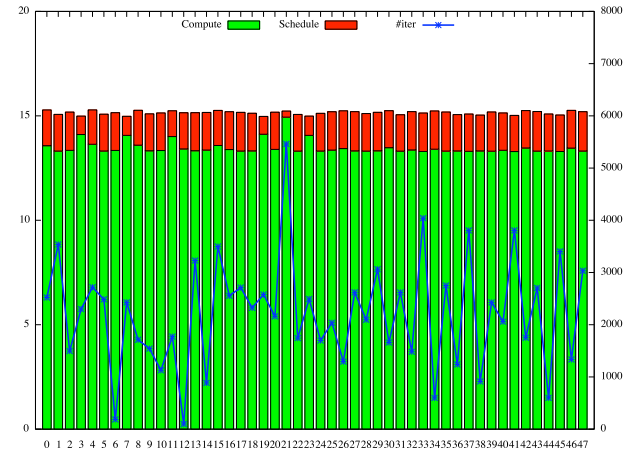

(b) Guided scheduler

Fig. 6: Times $(\mathrm{ms})$ per core for the same PMA iteration with dynamic and guided schedulers. The histograms have the same scale. 


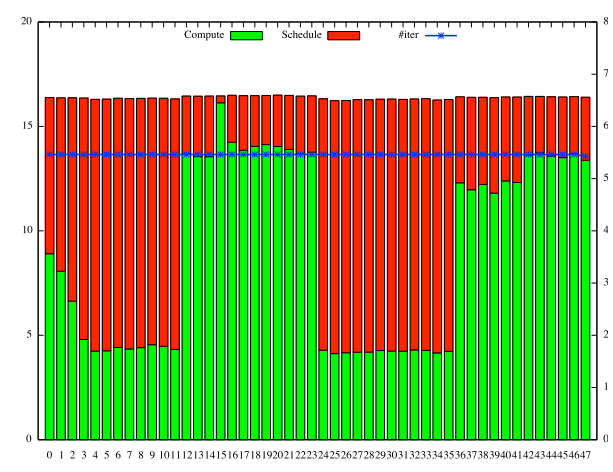

(a) Static scheduler

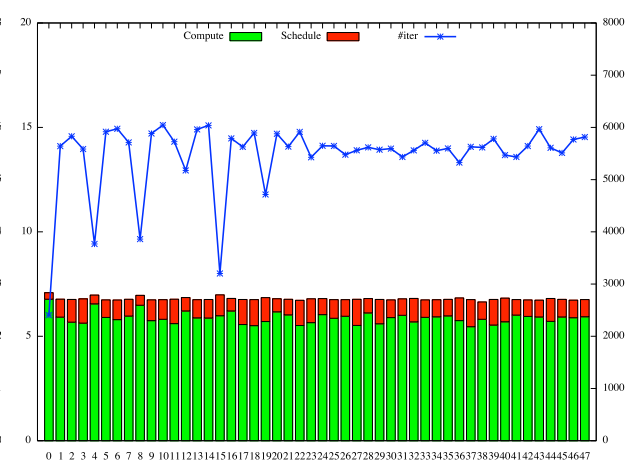

(b) Adaptive scheduler

Fig. 7: Times $(m s)$ per core for the same PAM iteration with static and adaptive schedulers. The histograms have the same scale.

The histogram represents the number of iterations performed by each core. We can conclude from the top two plots that:

1. the libGOMP dynamic scheduler with a chunk size of 1 spends a lot of time in the runtime system. This is mainly due to contention generated by concurrent accesses to internal data structures,

2. the number of iterations and the time spent executing iterations vary from one core to another. The computation of a correct chunk size helps decreasing the schedule time. The performance is thus increased as the average time per iteration decreases from $57.2 \mathrm{~ms}$ to $14.2 \mathrm{~ms}$.

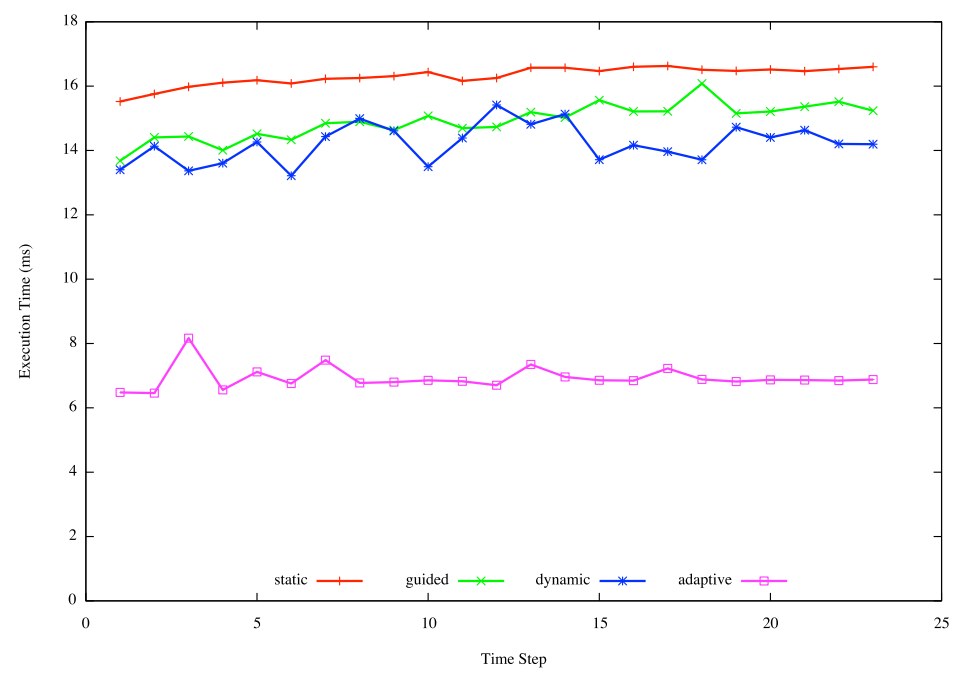

Fig. 8: Comparison of loop schedulers with respect to the time step of PMA simulation. 
Figures $7 \mathrm{a}$ and $7 \mathrm{~b}$ report results with the static and our adaptive loop schedulers with a bloc data distribution. The blue line in the bottom left plot validates the behavior of the static scheduler, as every thread executes the same number of iterations. Nevertheless, the CPU times are highly variable: the distribution of iterations fails at balancing the workload. On the other hand, our adaptive scheduler is able to keep the workload well balanced at the expense of an irregular distribution of iterations. The average execution time is $6.95 \mathrm{~ms}$ for the adaptive scheduler and $16.3 \mathrm{~ms}$ for the static scheduler.

Figure 8 reports the behavior of the 4 loop schedulers on PMA simulation with respect to the time step. Even if the iterations are well balanced among the cores, the static scheduler is unable to balance the workload. Both the dynamic and the guided schedulers reach the same level of performances and are able to better balance the workload, even if memory affinity is not ensured. Our adaptive scheduler is a good compromise between the static and the dynamic schedulers, and reaches a speed-up of 2.35 over the static scheduler.

\section{Conclusion and Future Work}

This paper introduced adaptive, a new OpenMP loop scheduler implementing a runtime-level approach to deal with irregular memory-bound applications on NUMA architectures. Instead of distributing statically-fixed portions of work over the threads of a parallel region, this scheduler adapts the granularity of work on demand by making idle threads steal a subset of the victim's remaining iterations, thus introducing the notion of dynamic per-thread granularity. Our scheduler is also capable of adapting its work-stealing algorithm to fit different memory bindings on NUMA architectures and outperforms OpenMP-based approaches to deal with memory locality, like the joint use of the static loop scheduling and the first-touch allocation policy, on several benchmarks and applications.

This work could be extended to task parallelism, providing the OpenMP application programmer with ways of annotating splitter functions called to generate parallelism on demand by splitting a running task into smaller ones. We also consider extending our approach using the concept of places recently added to the OpenMP standard that could help the programmer transmitting valuable and portable information on the way memory should be allocated on hierarchical architectures.

\section{Acknowledgement}

This work has been partially supported by the ANR 09-COSI-011-05 Project Repdyn and the FP7-PEOPLE-2011-IRSES Project HPC-GA (295217).

\section{References}

1. E. Ayguadé, B. Blainey, A. Duran, J. Labarta, F. Martínez, X. Martorell, and R. Silvera. Is the schedule clause really necessary in openmp? In Proceedings of the OpenMP applications and tools 2003 international conference on OpenMP shared memory parallel programming, WOMPAT'03, pages 147-159, Berlin, Heidelberg, 2003. Springer-Verlag. 
2. F. Broquedis, O. Aumage, B. Goglin, S. Thibault, P.-A. Wacrenier, and R. Namyst. Structuring the execution of OpenMP applications for multicore architectures. In Proceedings of 24th IEEE International Parallel and Distributed Processing Symposium (IPDPS'10), Atlanta, GA, April 2010. IEEE Computer Society Press.

3. F. Broquedis, T. Gautier, and V. Danjean. Libkomp, an efficient openmp runtime system for both fork-join and data flow paradigms. In Proceedings of the 8th international conference on OpenMP in a Heterogeneous World, IWOMP'12. Springer-Verlag, 2012.

4. F. Broquedis, N. Furmento, B. Goglin, R. Namyst, and P.-A. Wacrenier. Dynamic Task and Data Placement over NUMA Architectures: an OpenMP Runtime Perspective. In International Workshop on OpenMP (IWOMP), Dresden, Allemagne, 2009.

5. J. M. Bull. Measuring synchronisation and scheduling overheads in openmp. In In Proceedings of First European Workshop on OpenMP, pages 99-105, 1999.

6. S. Che, M. Boyer, J. Meng, D. Tarjan, J. W. Sheaffer, S.-H. Lee, and K. Skadron. Rodinia: A benchmark suite for heterogeneous computing. In Proceedings of the 2009 IEEE International Symposium on Workload Characterization (IISWC), IISWC '09, pages 44-54, Washington, DC, USA, 2009. IEEE Computer Society.

7. M. Durand, B. Raffin, and F. Faure. A Packed Memory Array to Keep Moving Particles Sorted. In 9th Workshop on Virtual Reality Interaction and Physical Simulation, 2012.

8. M. Frigo, C. E. Leiserson, and K. H. Randall. The implementation of the cilk-5 multithreaded language. SIGPLAN Not., 33(5):212-223, May 1998.

9. R. C. Hoetzlein. Fluids v2.0, open source, fluid simulator, 2008.

10. L. Huang, H. Jin, L. Yi, and B. Chapman. Enabling locality-aware computations in openmp. Sci. Program., 18(3-4):169-181, August 2010.

11. M. Ihmsen, N. Akinci, M. Becker, and M. Teschner. A parallel sph implementation on multicore cpus. Computer Graphics Forum, 30(1):99-112, 2011.

12. A. Mahéo, S. Koliaï, P. Carribault, M. Pérache, and W. Jalby. Adaptive openmp for large numa nodes. In Proceedings of the 8th international conference on OpenMP in a Heterogeneous World, IWOMP'12, pages 254-257, Berlin, Heidelberg, 2012. Springer-Verlag.

13. A. Marowka, Z. Liu, and B. Chapman. Openmp-oriented applications for distributed shared memory architectures: Research articles. Concurr. Comput. : Pract. Exper., 2004.

14. J. D. McCalpin. Memory bandwidth and machine balance in current high performance computers. IEEE Computer Society Technical Committee on Computer Architecture (TCCA) Newsletter, pages 19-25, December 1995.

15. S. L. Olivier, B. R. de Supinski, M. Schulz, and J. F. Prins. Characterizing and mitigating work time inflation in task parallel programs. In Proceedings of the International Conference on High Performance Computing, Networking, Storage and Analysis, SC '12, pages 65:165:12, Los Alamitos, CA, USA, 2012. IEEE Computer Society Press.

16. S. L. Olivier, A. K. Porterfield, K. B. Wheeler, M. Spiegel, and J. F. Prins. Openmp task scheduling strategies for multicore numa systems. Int. J. High Perform. Comput. Appl., 26(2):110-124, May 2012.

17. OpenMP Architecture Review Board. http://www.openmp.org, 1997-2008.

18. S. Subramaniam and D. L. Eager. Affinity scheduling of unbalanced workloads. In Proceedings of the 1994 ACM/IEEE conference on Supercomputing, Supercomputing '94, pages 214-226, Los Alamitos, CA, USA, 1994. IEEE Computer Society Press.

19. M. Tchiboukdjian, V. Danjean, T. Gautier, F. Le Mentec, and B. Raffin. A work stealing scheduler for parallel loops on shared cache multicores. In Proceedings of the 2010 conference on Parallel processing, Euro-Par 2010, pages 99-107. Springer-Verlag, 2011.

20. D. Traoré, J.-L. Roch, N. Maillard, T. Gautier, and J. Bernard. Deque-free work-optimal parallel stl algorithms. In Proceedings of the 14th international Euro-Par conference on Parallel Processing, Euro-Par '08, pages 887-897, Berlin, Heidelberg, 2008. 
21. Y. Yan, C. Jin, and X. Zhang. Adaptively scheduling parallel loops in distributed sharedmemory systems. IEEE Trans. on Parallel and Distributed Systems, 8(1), January 1997. 